\title{
A Study on the Field Strength Prediction of a Ground-wave Based Time Broadcasting Transmitter Station in the Korean Peninsula
}

\author{
Sun Yong Lee', Yun Sub Choi', Sang-wook Hwang', Sung-hoon Yang², Chang-Bok Lee², Sang Jeong Lee ${ }^{1 \dagger}$ \\ ${ }^{1}$ Department of Electronics Engineering, Chungnam National University, Daejeon 305-764, Korea \\ ${ }^{2}$ Korea Research Institute of Standards and Science, Daejeon 305-340, Korea
}

\begin{abstract}
In this study, to improve an existing ground-wave based time broadcasting system, a study that predicts the field distribution and field strength of the transmitted signal of a new ground-wave based time broadcasting system was performed. The prediction area was assumed to be the Korean peninsula; and to reflect the mountainous terrain of the Korean peninsula in the prediction of the variations of field distribution and field strength, a new prediction method based on the Monteath model was proposed and utilized. As field distribution changes depending on the position of a transmitter station, potential sites for the transmitter station were selected considering the geographical characteristics. In this regard, the ground conductivity information of North Korea cannot be obtained, and thus, the ground conductivity of the North Korean region was reflected considering the geological characteristics of South Korea and North Korea. Based on this, the variations of field distribution and field strength were predicted by setting the Korean peninsula as the prediction area, and the prediction results depending on the position of the transmitter station were discussed.
\end{abstract}

Keywords: ground-wave, Monteath, field attenuation

\section{INTRODUCTION}

Time information is important information that is used for basic national infrastructures, and it is provided from GPS or HLA. Time information is mostly provided from GPS. However, GPS signals are vulnerable to jamming, and in case of emergency, GPS-based time information cannot be used. Thus, it needs to be substituted by ground-wave based time information. Currently, in South Korea, the groundwave based time broadcasting system, HLA, is operated by the Korea Research Institute of Standards and Science. HLA provides time information using the frequency band with a center frequency of $5 \mathrm{MHz}$ at a transmitted power of $2 \mathrm{~kW}$. For HLA, a number of shadow zones that time information cannot reach occur in South Korea, and thus, the use of

Received Apr 09, 2014 Revised May 12, 2014 Accepted May 15, 2014 †Corresponding Author

E-mail: eesjl@cnu.ac.kr

Tel: +82-42-825-3991 Fax: +82-42-823-5436 the time information of HLA is also limited. In general, the propagation loss of ground waves increases as the frequency increases. To stably and universally apply time information to basic national infrastructures, a new time broadcasting system that minimizes shadow zones by lowering the frequency bands of time information signals needs to be established. In the case of the design for the establishment of a new time broadcasting system, design details for system operation should be considered. However, in this study, to use time information before design, the premise was the prediction of minimum field strength in the application area compared to the transmitted power.

The signal frequency band of a new time broadcasting system is different from that of an existing time broadcasting system. Thus, studies on a new time broadcasting system need to be based on simulation. The examples of a groundwave based time broadcasting system in foreign countries include DCF77 from Germany, JJY from Japan, WWVB from the United States, and RTZ from Russia (Kuhn 2006). The DCF77 from Germany uses a transmitted power of 50 $\mathrm{kW}$ and a frequency of $77.5 \mathrm{kHz}$ (Bauch et al. 2009), the JJY 
from Japan uses $50 \mathrm{~kW}$ and $40 \mathrm{kHz} / 60 \mathrm{kHz}$ (JSTG 2005), and the WWVB from the United States uses $50 \mathrm{~kW}$ and $60 \mathrm{kHz}$. For these systems, to predict the variations of the field distribution and field strength of time information signals during research, a prediction method based on the Millington model recommended by ITU was used (Millington 1949, ITU 1992). This method has been used in numerous studies, and is regarded as a typical method. The present study examines a time broadcasting system for the Korean peninsula, and the Korean peninsula is small compared to other countries and has mountainous terrain. Thus, the effect of mountainous terrain, which has not been considered in the systems from other countries, should be considered. The prediction method based on the Millington model, which was typically used in the research of the time broadcasting systems from other countries, cannot reflect terrain elevation, and thus, there is a difficulty in reflecting the effect of mountainous terrain. Therefore, in this study, a new prediction method based on the Monteath model, which reflects the terrain elevation of prediction area, was proposed and utilized. In this regard, the time information application area was assumed to the Korean peninsula including the North Korean region, rather than considering only the South Korean region, because including the North Korean region is thought to be a rational choice considering the future. Mountainous terrain was considered because it is related with the occurrence of shadow zones. The Monteath model can examine the information on field strength and propagation delay at the same time. Propagation delay is important information for a time broadcasting system. However, in this study, a propagation delay element was not considered because this study is a study that predicts the variations of the field distribution and field strength of time information signals for application area and because propagation delay presumes that time information signals can be received.

For studies on a new ground-wave based time broadcasting system, to minimize shadow zones, the frequency band of a time information signal can be lowered, the transmitted power can be increased, or potential sites for the transmitter station can be selected considering the geographical characteristics. In this regard, potential sites for the transmitter station consider areas where the establishment of a time broadcasting transmitter station is possible.

Ground conductivity is reflected during the prediction simulation of field distribution and field strength. As the ground conductivity of the North Korean region cannot be obtained, the minimum value of the ground conductivity of the South Korean region was used. This is because the rock distribution or composition of the ground is not significantly different between South Korea and North Korea and because conductivity is determined by the moisture content of the rock of ground surface rather than by geological composition (McNeill 1980). Also, conductivity changes due to the effect of climate, and thus, accurate calculation is difficult. In this study, the emphasis is on the method for minimizing shadow zones. Therefore, to derive the prediction results of the worst case, the minimum value of the ground conductivity of South Korea was used as the ground conductivity of North Korea.

In this study, to improve an existing ground-wave based time broadcasting system, a study that predicts the field distribution and field strength of the transmitted signal of a new ground-wave based time broadcasting system was performed. The prediction area was assumed to be the Korean peninsula; and to reflect the mountainous terrain of the Korean peninsula in the prediction of the variations of field distribution and field strength, a new prediction method based on the Monteath model was proposed and utilized. As field distribution changes depending on the position of a transmitter station, potential sites for the transmitter station were selected considering the geographical characteristics. In this regard, the ground conductivity information of North Korea cannot be obtained, and thus, the ground conductivity of the North Korean region was reflected considering the geological characteristics of South Korea and North Korea. Based on this, the variations of field distribution and field strength were predicted by setting the Korean peninsula as the prediction area, and the prediction results depending on the position of the transmitter station were discussed.

\section{FIELD STRENGTH PREDICTION MODEL}

To predict the field strength of the ground waves transmitted from a ground-wave based time broadcasting system, a new prediction method based on the Monteath model was used. For the proposed prediction method based on the Monteath model, two kinds of models are used. One is the model that has been organized as a function of distance when the propagation environment is assumed to be a free space, and it predicts field strength using the distance between a transmitter station and a receiving point in a free space (ITU 1994). The other is the Monteath model, and it predicts field strength depending on the distance and also predicts additional field strength attenuation using the ground conductivity and terrain elevation on the propagation path (Monteath 1978). Therefore, the 
prediction method based on the Monteath model predicts the field strength at a receiving point by adding each result value of the two models.

The Monteath model predicts additional field strength attenuation using the value of the complex attenuation factor, $G$. In this regard, the propagation path of the complex attenuation factor defined using an integral equation is divided into regular intervals, and the complex attenuation factor is expressed as the sum of integration, as shown in Eq. (1) (Monteath 1978).

$$
G(N D)=\frac{1-B N^{1 / 2} D^{1 / 2} \sum_{I=0}^{N-1} f(I) f(N-I) K(N, I) G(I D) E(N D, I D)}{1+B D^{1 / 2} K(N, N) E(N D, N D)}
$$

where $G$ is the complex attenuation factor, $D$ is the path interval, $N$ is the number of the sections obtained through dividing the propagation path by the path interval, $I$ is the number of the sections obtained through dividing the propagation path by the path interval at the center point of the propagation path, $K$ is the coefficient, and $E$ is the effective ground impedance. $B$ is expressed in Eq. (2), and $f$ $(m)$ is expressed in Eq. (3).

$$
\begin{aligned}
B & =\sqrt{\frac{j}{\lambda}} \\
f(m) & = \begin{cases}1 & \text { if } m=0 \\
m^{-\frac{1}{2}} & \text { if } m>0\end{cases}
\end{aligned}
$$

where $j$ is $\sqrt{-1}$, and $\lambda$ is the wavelength in a free space.
The differences in the prediction method based on the Monteath model and the prediction method based on the Millington model were compared. The two prediction methods have the same application purpose in predicting field strength, but have differences in the prediction results depending on the reflection of an environment variable. For the prediction method based on the Monteath model, the effect of terrain elevation is reflected; whereas for the prediction method based on the Millington model, the effect of terrain elevation is not reflected. Fig. 1 shows the prediction results depending on each prediction method. In this study, the prediction method based on the Monteath model, which reflects the effect of terrain elevation, was used.

\section{PREDICTION PLATFORM}

The prediction method based on the Monteath model for field strength prediction is implemented in a prediction simulation platform, and performs prediction. In the prediction simulation platform, the field strength of the transmitted signal at each receiving point is predicted using the conductivity and elevation information of a prediction area. During field strength prediction, receivers that are arranged in a regular lattice form in the prediction area are assumed, and the ground-wave field strength at each receiving point is predicted through the field strength prediction model. Using the field strength prediction value at each receiving point, a field strength prediction map for the prediction area is generated; and based on the generated
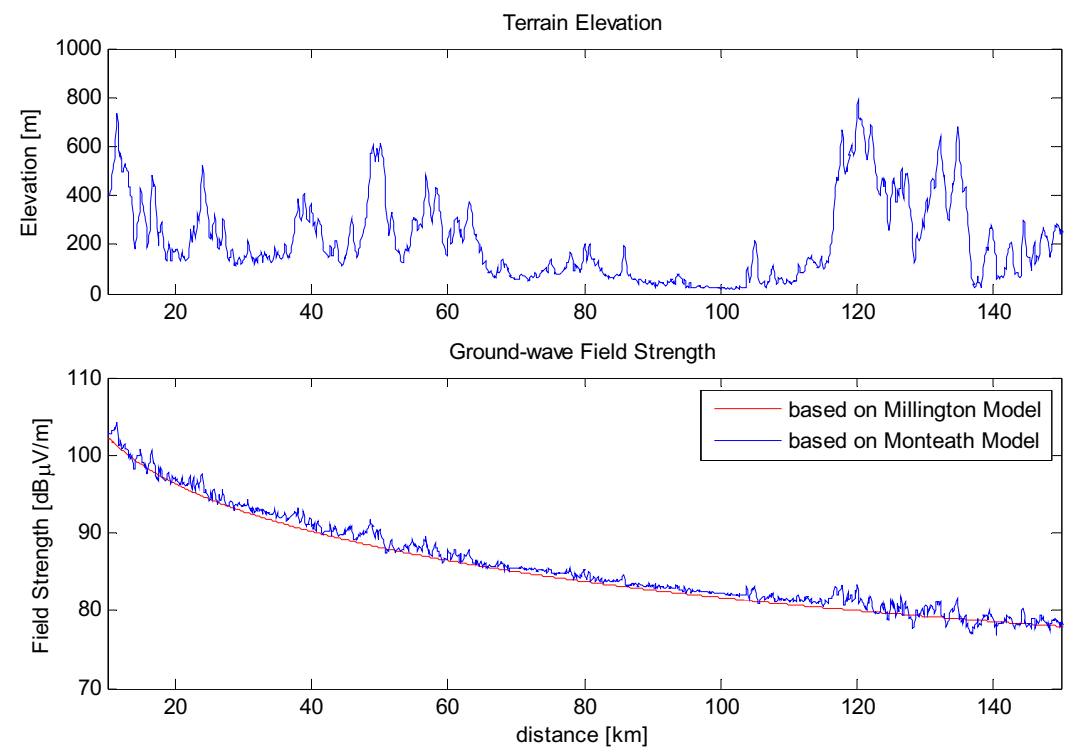

Fig. 1. Compares the field strength prediction models. 
field strength prediction map, the field strength distribution of the ground waves transmitted from a transmitter station is predicted. The position of the transmitter station is considered because the field strength prediction map shows different field strength distribution results depending on the position of the transmitter station. To perform field strength prediction, conductivity and elevation data are processed considering the position of a transmitter station and a receiving point, and field strength prediction values are calculated using the processed data and the field strength prediction model. The processes from data to field strength prediction are handled by dividing them into a preprocessing module and a prediction model processing module.

\subsection{Preprocessing Module}

The field strength at a receiving point depending on the desired position of a transmitter station is predicted through the field strength prediction model. In this regard, the field strength prediction model requires the ground conductivity data and terrain elevation data on the propagation path that reflected the position of a transmitter station and a receiving point. For the ground conductivity data to be used in the prediction model, the conductivity information of South Korea can be obtained through the atlas ground conductivity data of Rec. ITU-R P832-3 from ITU (ITU 2012); and for the terrain elevation data to be used in the prediction model, the elevation data of the Korean peninsula and the adjacent area can be obtained through the Shuttle Radar Topography Mission (NASA SRTM) (NASA 2000). Fig. 2 shows the ground conductivity data obtained from the atlas ground conductivity data of Rec. ITU-R P8323 from ITU and the terrain elevation data obtained from NASA SRTM.

The obtained ground conductivity data and terrain elevation data are in the form of a map. Thus, the preprocessing module processes the ground conductivity data and terrain elevation data in the form of linear-type data by reflecting the position of a transmitter station and a receiving point in each obtained data. Also, as the Monteath model adds in the form of progression, the path interval of the propagation path is also reflected during the data processing. Fig. 3 shows the flow chart of the preprocessing module. For the processing of the conductivity data and the elevation data, the sizes of the two data maps are set to 1:1 ratio, and they are processed as the ground conductivity and terrain elevation data on a linear-type propagation path.

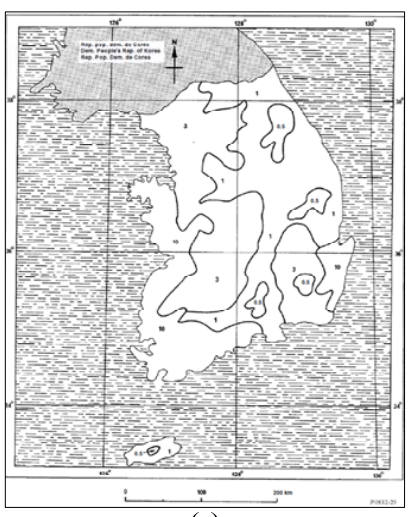

(a)

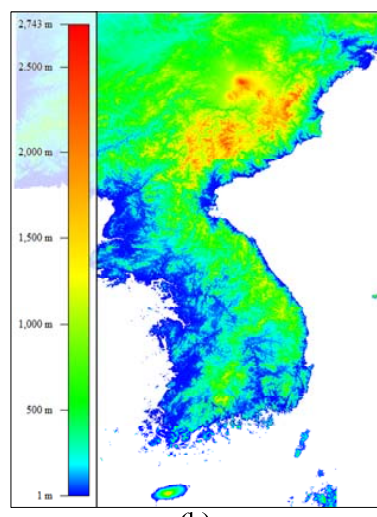

(b)
Fig. 2. Raw data: (a) Conductivity data and (b) Elevation data.

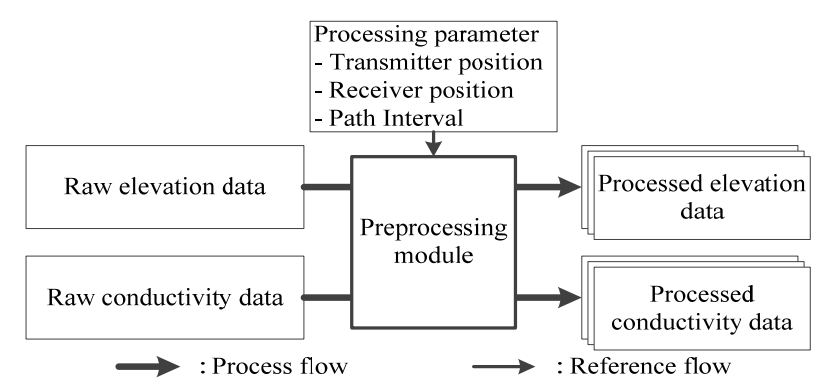

Fig. 3. Flow chart of preprocessing module

\subsection{Prediction Model Processing Module}

In the prediction model processing module, the field strength at a receiving point is predicted using the field strength prediction model that applies the prediction method based on the Monteath model. In this regard, the field strength at a receiving point is predicted by applying the ground conductivity data and terrain elevation data processed in the preprocessing part to the field strength prediction model. The field strength at a receiving point is derived by adding the field strength prediction result in a free space and the additional field strength attenuation prediction result obtained by the Monteath model. The field strength prediction value for a receiving point is derived at each receiving point of the prediction area; and if the field strength prediction values for each receiving point are gathered together, the field strength distribution depending on the corresponding transmitter station is obtained in the form of a map. In this regard, the field strength in a free space is derived by reflecting the distance between a transmitter station and a receiving point, and the additional field strength attenuation due to the ground conductivity and terrain elevation is predicted by the Monteath model. Fig. 4 shows the flow chart of the implemented algorithm processing module. 


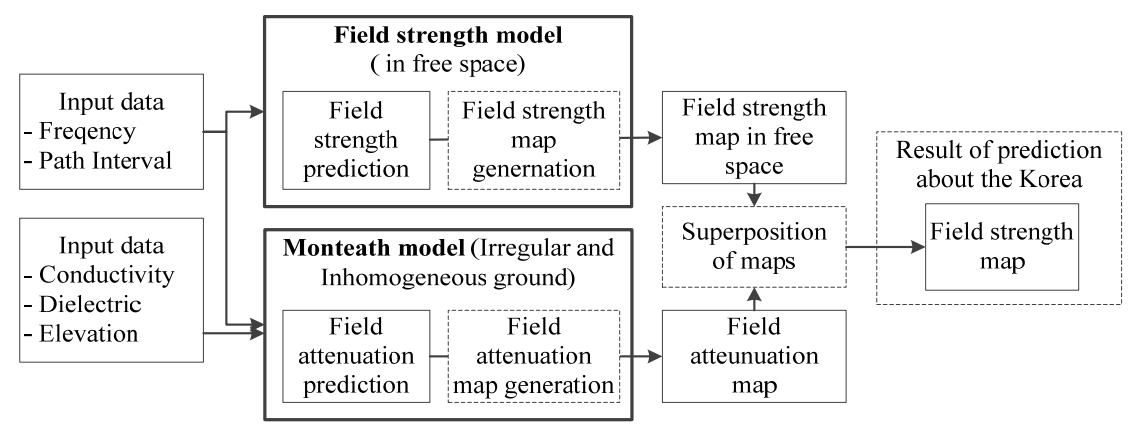

Fig. 4. Flow chart of processing module of prediction model.

\section{PREDICTION ENVIRONMENT}

For the prediction simulation platform, to predict the field distribution of the new ground-wave based time broadcasting system, environment variables relevant to the new time broadcasting system need to be established. For the time information signal of the new time broadcasting system, the frequency of the time information signal was set to $70 \mathrm{kHz}$, which is a low frequency band with good propagation characteristics, and the transmitted power was set to $50 \mathrm{~kW}$, considering the various examples from foreign countries. The time information application area of the new time broadcasting system was assumed to be the Korean peninsula. Thus, the longitude of the prediction area was set to $124.3^{\circ} \sim 131.0^{\circ}$, and the latitude was set to $33.1^{\circ} \sim$ $43.0^{\circ}$. To regularly arrange receiving points in the prediction area, it was set to $10 \times 10 \mathrm{~km}$ lattice size. The Monteath model used for field strength prediction calculates field strength at a constant path interval of the propagation path. In this regard, the path interval is related with the accuracy of calculation, and it was set to $100 \mathrm{~m}$ for sufficiently small calculation error. In the case of the ground conductivity that is closely related with propagation loss, $5 \mathrm{~S} / \mathrm{m}$ was used for the sea surface, and the atlas ground conductivity data provided by the Rec. ITU-R P832-3 of ITU was used for the South Korean region. In the case of the North Korean region,

Table 1. Parameters of platform environment.

\begin{tabular}{llc}
\multicolumn{1}{c}{ Type } & Value \\
\hline Frequency $(\mathrm{kHz})$ & & 70 \\
Transmitted Power $(\mathrm{kW})$ & & 50 \\
Path interval $(\mathrm{m})$ & Sea level & 100 \\
& The South Korea & Rec. ITU-R P832-3 data \\
Conductivity $(\mathrm{mS} / \mathrm{m})$ & Other region & 0.5 \\
& & NASA SRTM data \\
Elevation & & 4 \\
Dielectric constant & & $33.1 \sim 43.0$ (Latitude) \\
& & $124.3 \sim 131.0$ (Longitude) \\
Prediction range $\left(^{\circ}\right)$ & & $10 \times 10$ \\
Lattice size $(\mathrm{km})$ &
\end{tabular}

the geological composition of the ground is not significantly different from that of South Korea. Thus, to derive the field strength variation of the worst case during the field strength prediction, $0.5 \mathrm{mS} / \mathrm{m}$, which is the lowest ground conductivity of South Korea, was used. For the terrain elevation, NASA SRTM data was used, and the dielectric constant was set to 4 . Table 1 summarizes the environment variables of the prediction simulation platform.

When the field strength of the prediction area is predicted, the position of the transmitter station should be considered. This is because the conductivity and elevation information of the prediction area is not changed during the performance of a prediction simulation, but each propagation path between the transmitter station and each receiving point depending on the position of the transmitter station changes and the conductivity and elevation information on the corresponding propagation path also changes. Therefore, the position of the transmitter station should be considered when the field strength of the prediction area is predicted. As the prediction area was the Korean peninsula, potential sites for the transmitter station were selected at the center of the Korean peninsula considering the geographical characteristics. For the geographical characteristics, a mountainous terrain, a gentle terrain, and an intermediate terrain were considered. Accordingly, Hyangnobong was selected as the mountainous terrain at the center of the Korean peninsula, Yeonpyeong Island was selected as the gentle terrain, and Cheorwon-eup was selected as the intermediate terrain of a mountainous terrain and a gentle terrain. For an existing time broadcasting system, a number of shadow zones currently occur in the South Korean region. Thus, to derive the field strength distribution of time information signals when only the South Korean region was considered, Mt. Songni, which is the center of South Korea, was additionally selected. Fig. 5 shows the position of each selected transmitter station. 


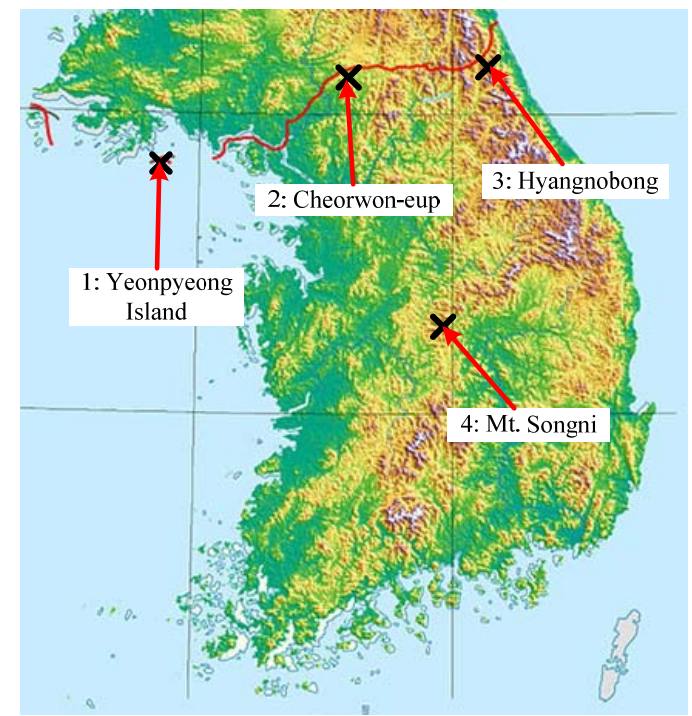

Fig. 5. Positions of the selected transmitter.

\section{PREDICTION RESULTS}

By setting the environment variables for the new time broadcasting system, the field distribution of time information signals in the Korean peninsula region was predicted. Based on the field distribution prediction results of time information signals, the field strength of the time information signals, transmitted from the transmitter station, at each receiving point of the time information application area can be analyzed, and the effects of the propagation environment factors on the field strength variation of the time information signals when the signals propagate on land and on the sea, respectively, can be analyzed. Fig. 6 shows the field distribution prediction results of the time information signals for each transmitter station.

First, common results are analyzed for the four kinds of prediction simulation results. In the above prediction simulation results, the field strength attenuation rate of low frequency band time information signals can be divided into the land environment and the sea surface environment. In general, field strength attenuation rate is high in the land environment with low conductivity, and is low in the sea surface environment with high conductivity. In utilizing time information, the field strength attenuation rate is related with the coverage of time information signals. For the section with high field strength attenuation rate, the coverage of time information signals is short, and thus, time information can be used only in the region that is close to the time broadcasting transmitter station. For the section with low field strength attenuation rate, the coverage of time information signals is long, and thus, time information can be used in the region that is relatively far from the time broadcasting transmitter station. When low frequency

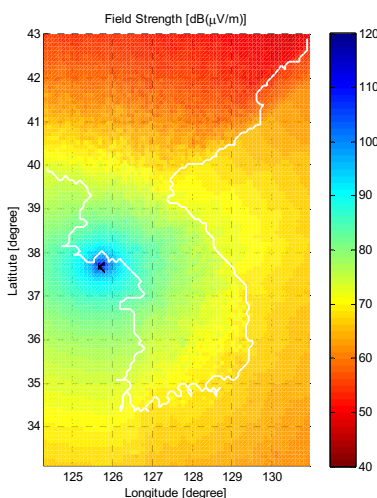

(a)

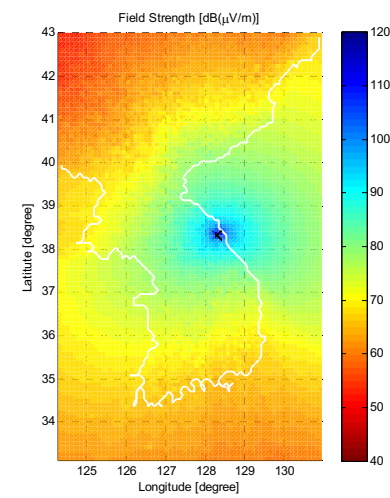

(c)

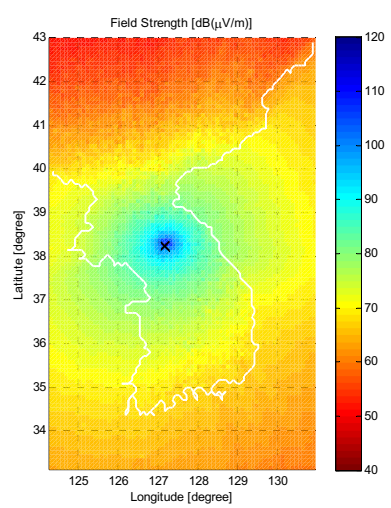

(b)

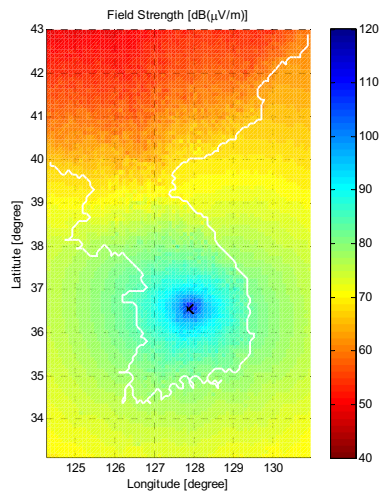

(d)
Fig. 6. Field strength prediction map corresponding to each transmitters, (a) Yeonpyeong Island, (b) Cheorwon-eup, (c) Hyangnobong, (d) Mt. Songni.

band time information signals pass mountainous terrain, the time information application area is affected by the variation of the terrain elevation, besides the effect of the conductivity of the propagation path. The results indicated that the field strength attenuation rate was not constant and showed a large deviation when the time information signals passed mountainous terrain. For this result, the position of the transmitter station could be important, but it could be regarded as a problem related with transmitted power. When the transmitted power is low, field strength variation is large in mountainous terrain, and thus, a number of shadow zones could occur. In contrast, when the transmitted power is high, the field strength of time information signals could be sufficiently large compared to the amount of attenuation despite mountainous terrain, and thus, shadow zones would decrease.

The prediction results for each potential site were analyzed. The time information application area was assumed to be the Korean peninsula, and the positions of the potential sites for the transmitter station were selected to be Yeonpyeong Island, Cheorwon-eup, and Hyangnobong. Time information signals were transmitted from the transmitter station at $50 \mathrm{~kW}$. The field strength distribution prediction simulation for these three potential sites indicated that the minimum field strength prediction 
result values were about $48 \mathrm{~dB}(\mu V / m), 55 \mathrm{~dB}(\mu V / m)$, and $60 \mathrm{~dB}(\mu V / m)$ when the transmitter station was positioned at Yeonpyeong Island, Cheorwon-eup, and Hyangnobong, respectively. The minimum field strength prediction result is the result obtained by the large field strength attenuation of time information signals when the time information signals propagate on a long propagation path. Accordingly, the field strength prediction result value of the time information signals at the northern end of Hamgyeongbuk-do, which is far from each transmitter station, becomes the minimum field strength prediction result value depending on the potential sites for the transmitter station. Especially, when the transmitter station was positioned at Hyangnobong, the northern and southern ends of the Korean peninsula had similar propagation distances from the transmitter station, and thus, similar sizes of field strength attenuation were observed at any boundary regions of the Korean peninsula with similar propagation paths. It can be interpreted that the field of the time information signals was evenly distributed in the Korean peninsula region because the position of the transmitter station was close to the center of the smallest circle that includes the Korean peninsula.

In this study, the validity of the field strength prediction results was examined using the values provided by JSTG from Japan. In using the examples from Japan, it was based on the distance between a transmitter station and a receiving point. In this study, the distances between Hamgyeongbuk-do and each potential site for the transmitter station were about $700 \mathrm{~km}$ for Yeonpyeong Island, about $580 \mathrm{~km}$ for Cheorwon-eup, and about $520 \mathrm{~km}$ for Hyangnobong. In the case of the field strength prediction values from Japan, the field strength prediction values at receiving points that are about $510 \mathrm{~km}, 600 \mathrm{~km}$, and $680 \mathrm{~km}$ apart from the Hagane-yama transmitter station in Japan were used. The average field strength prediction values at the points that are about $510 \mathrm{~km}, 600 \mathrm{~km}$, and $680 \mathrm{~km}$ apart from the Hagane-yama transmitter station were 65 $\mathrm{dB}(\mu V / m), 66 \mathrm{~dB}(\mu V / m)$, and $61 \mathrm{~dB}(\mu V / m)$, respectively (JSTG 2005). When the prediction results of this study and the prediction values from Japan were compared based on the field strength prediction values at similar distances, respectively, they showed significant differences. However, it should be considered that the differences between the results of this study and the prediction values from Japan were observed because in the case of Japan, a prediction method based on the Millington model was used and the carrier frequency of the time information signal was $60 \mathrm{kHz}$; while in this study, the lowest conductivity was uniformly applied to the region other than South Korea. Therefore, when the field strength prediction result of this study and the field strength prediction result from Japan are compared, it is thought that the field strength prediction result of this study is valid.

Lastly, when the time information application area was assumed to be South Korea, the potential site for the transmitter station was selected to be Mt. Songni. The time information signals were transmitted at $50 \mathrm{~kW}$, and the minimum field strength prediction result value in the South Korean region was about $72 \mathrm{~dB}(\mu \mathrm{V} / \mathrm{m})$. When the potential site for the transmitter station was positioned at Mt. Songni, the time information application area was small compared to the transmitted power, and the deviation of the field strength attenuation was not large as the signals propagated. Therefore, it is thought that the occurrence of shadow zones due to mountainous terrain would be scarce.

\section{CONCLUSIONS}

In this study, to improve an existing ground-wave based time broadcasting system, a study that predicts the field distribution and field strength of the transmitted signal of a new ground-wave based time broadcasting system was performed. In the case of the prediction method for the field distribution and field strength of time information signals, a prediction method based on the Monteath model, which derives prediction results by reflecting the terrain elevation of a prediction area, was used. A prediction simulation was performed by applying the environment variables of the new time broadcasting system to a prediction platform based on the Monteath model. The results indicated that when the time information application area was assumed to be the Korean peninsula, among the minimum field strength prediction values of the prediction area for each transmitting point, Hyangnobong showed the highest value $(60 \mathrm{~dB}(\mu V / m))$. This result can be interpreted that the field of the time information signals transmitted from Hyangnobong was evenly distributed in the Korean peninsula region compared to other transmitter stations. When the result of this study is compared with the field strength prediction result values from Japan, it is thought that the field strength prediction result values of this study are valid. When the time information application area was assumed to be South Korea, the minimum field strength prediction value was $72 \mathrm{~dB}(\mu \mathrm{V} / \mathrm{m})$. The results and analysis indicated that when the new time broadcasting system was established assuming the Korean peninsula as the time information application area, the Hyangnobong region was the appropriate potential site for the transmitter station. When the new time broadcasting system was established for the South Korean region, the field strength of the time information signals generally increased, and the deviation of the field strength attenuation was not large as the signals propagated in mountainous terrain. Therefore, it is thought that the occurrence frequency of shadow zones due to mountainous terrain would decrease. Lastly, this study could provide the design indices of a new time broadcasting system design by selecting a new potential site for the transmitter station and by deriving the minimum field strength that can be received compared to the transmitted power of the new time broadcasting system. 


\section{ACKNOWLEDGMENTS}

This research was supported by Basic Science Research Program through the Korea Research Institute of Standards and Science (KRISS) of Korea funded by the Ministry of Education, Science and Technology.

\section{REFERENCES}

Bauch, A., Hetzel, P., \& Piester, D. 2009, Time and Frequency Dissemination with DCF77: From 1959 to 2009 and beyond, PTB mitteilungen, 119 (3)

ITU 1992, Ground-wave Propagation Curves for Frequencies between $10 \mathrm{kHz}$ and $30 \mathrm{MHz}$, ITU-R P.368-7 Recommendation

ITU 1994, Calculation of Free-space Attenuation, ITU-R P.525-2 Recommendation

ITU 2012, World atlas of ground conductivities, ITU-R P.8323 Recommendation

JSTG 2005, JJY [Internet], available from: http://jjy.nict. go.jp/jjy/trans/index-e.html

Kuhn, M. 2006, Low-frequency Radio Time Signal [Internet], available from: https://www.cl.cam.ac.uk/ mgk25/ time/lf-clocks/

McNeill, J. D. 1980, Electrical Conductivity of Soils and Rocks, Geonics Limited Technical Note TN-5 [Internet], available from: http://www.georentals.co.uk/tn5.pdf

Millington, G. 1949, Ground-wave Propagation Over an In-homogeneous Smooth Earth, Proc. IEE, 96, 53-64. http://dx.doi.org/10.1049/pi-3.1949.0013

Monteath, G. D. 1978, Computation of ground-wave attenuation over irregular and inhomogeneous ground at low and medium frequencies, BBC Research Reports, BBC RD 1978/7

NASA 2000, SRTM [Internet], cited 2009 June 17, available from: http://www2.jpl.nasa.gov/srtm/

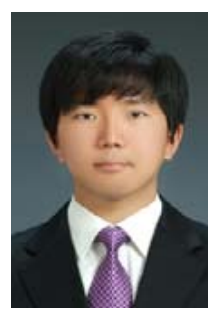

Sun Yong Lee received the Bachelor of degree in Electronics from Chungnam National University in 2013. His research interests include GNSS receivers and signal processing techniques.

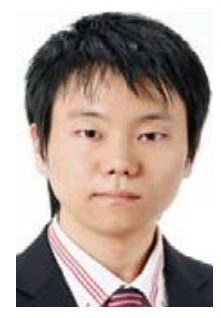

Yun Sub Choi received the Bachelor of degree in Electronics from Chungnam National University in 2010. His research interests include GNSS receivers and antijamming techniques.

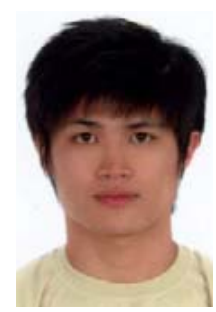

Sang-wook Hwang received the Master's degree in Electronics from Chungnam National University in 2010. His research interests include anti-jamming techniques and GNSS SDR.

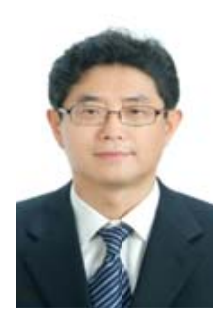

Sung-hoon Yang received the Doctor's degree in Control in Chungnam National University in 2012. His research interests include time synchronization and time transfer.

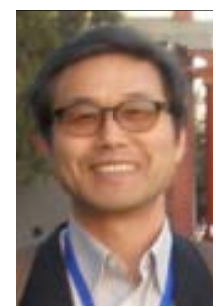

Chang-Bok Lee received the Doctor's degree in Microwave Engineering in Sogang University in 1984. His research interests include time transfer and GNSS.

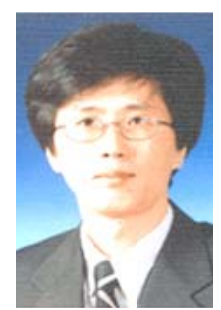

Sang Jeong Lee received the Doctor's degree in Control and Measurement in Seoul National University in 1987. His research interests include GNSS and Robust Control. 